Usage, Usability, and Utility of 3D City Models, 03006 (2012)

DOI: $10.1051 / 3 \mathrm{u} 3 \mathrm{~d} / 201203006$

(C) Owned by the authors, published by EDP Sciences, 2012

\title{
Integration of urban knowledge through visibility studies and pedestrian behavior analysis in Old Bazaar in Skopje
}

\author{
O. Marina ${ }^{1}$, T. Leduc ${ }^{2}$, G. Rabino ${ }^{3}$, S. Gievska ${ }^{4}$ and B. Karanakov ${ }^{1}$ \\ ${ }^{1}$ Faculty of Architecture, University Sts. Cyril and Methodius in Skopje, Macedonia \\ 2 LUNAM Université, CNRS, CERMA UMR 1563, École Nationale Supérieure \\ d'Architecture de Nantes, France \\ ${ }^{3}$ Department of Architecture and Planning, Politecnico di Milano, Italy \\ ${ }^{4}$ Faculty of Computer Science and Engineering, University Sts. Cyril and \\ Methodius in Skopje, Macedonia
}

\begin{abstract}
The series of studies that are focused on the Old Bazaar - the historic and protected district in city of Skopje, exercise the complex spatial analysis or the urban environment with use of models of visibility, patterns of spatial configuration and pedestrian behavior as scientific knowledge and research tools for better understanding of the processes that are shaping our cities. An integrated knowledge base has been proposed as the basis for urban models, being the container of a variety of traceable information needed for exhaustive description of the urban context that can provide a scientific platform for more sustainable management and development of urban protected areas.
\end{abstract}

\section{INTRODUCTION}

Programs for preservation of the historic district in Skopje have led to a separation and isolation through fixing in time the physical structure of the city. This approach does not recognize the distinctive dynamics of the way the urban space and architecture are perceived and used, especially by the pedestrians. Better understanding of the emerging patterns of pedestrian behavior within the urban environment and their interaction with the built environment can help us become more sensible to the processes that are occurring in the protected areas of the cities and especially within the historic districts that are frequently proclaimed pedestrian areas.

The motivation behind this work is the belief that effective and useful urban models cannot be accomplished without proper knowledge base that integrates knowledge in a meaningful way in a variety of formats. Use of appropriate analytical tools for deciphering a corresponding level of human activity and perception in a 3D urban environment is essential for establishing a minimum level of correspondence between the newly produced categories of knowledge. An integrated knowledge base has been proposed as the basis for urban models, being the container of a variety of traceable information needed for exhaustive description of the urban context. By organizing theoretically-and empirically-sound knowledge for enriched representation of urban living in a coherent framework, we have attempted to improve the methodology of the modeling process.

The purpose of this research is to investigate how pedestrian mobility data from Skopje' historic district and especially how the pedestrian perception can be visualized and presented in multilayered model that will provide cohesion and interaction between two or more aspects of this activity within the urban environment and to suggest how this novel information can be used for evaluation and better design of urban protected areas. This paper concludes with ideas how using the multilayered

This is an Open Access article distributed under the terms of the Creative Commons Attribution License 2.0, which permits unrestricted use, distribution, and reproduction in any medium, provided the original work is properly cited. 
and comparative model of pedestrian mobility for representing complex data and different variables of the urban environment could generate a result that would elucidate the complexity of the urban dynamics and would serve as a valuable tool for architects, planners, local authorities, citizens and even retailers in their effort to create more livable and human urban environment.

\section{INTEGRATING URBAN KNOWLEDGE}

People do not move in cities for mysterious reasons, they move because of the activity they are engaged in. These activities occur in urban environment that is defined by its attributes; physical like their morphology or less physical like configuration. Therefore, pedestrian mobility is always a function of activities and these environmental attributes. Measuring and modeling spatial aspects of the urban environment is therefore vitally related to the spatial cognition of pedestrians of the urban environment. The integration of different approaches that we intend to use is due to the necessity first, to represent the complexity of the urban environment and pedestrian mobility and second, to build a comprehensive platform for integration of the gained knowledge. Following the idea that the human spatial behavior cannot be investigate detached from the shape and configuration of the built environment we have tried to perform spatial, visibility and configurational analysis of the Old Bazaar in Skopje and to integrate and evaluate generated information through the agent modeling process. The correlation of the different studies has been proposed due to their focus on local scale of the built environment and the anthropocentric prospective. Finally, we argue that the possible enrichment of 3D city models with integrated urban knowledge of morphological, visual and configurational analysis of the built environment and pedestrian behavior could be of benefit for management of pedestrian traffic in urban protected areas through spatial navigation and space use and especially in evaluation of future urban development projects.

\subsection{Pedestrian mobility}

The pedestrian behavior can be described at three major levels: strategic level, tactical level, and operational level [1]. At the strategic level, the pedestrian decide what activity they will perform and the activity order. This level is generally comprehended even before arriving at the pedestrian area and therefore is considered exogenous for the models of simulation of pedestrian behavior. The tactical level is an expression of short-term decisions of the pedestrians regarding the movement within the urban area. While on the strategic level pedestrian have determined the list of the activities they plan to perform, on the tactical level pedestrians decipher: the order or schedule of activities; the choice of the activity area, and; the choice of the route. This level introduces the plan of sequence of activities and the timeframe of each activity, as well as the spatial area or the place and the route of the activity. At the operational level pedestrians make instantaneous decisions for the immediate time period and the built environment around them whether it is based on its physical or configurational aspects. Decisions made on this level are recognized as the walking behavior of the pedestrians. This level brings up the interaction between the activity of the person, its spatial perception and its interaction with the urban environment.

Different levels of pedestrian activity are connected with different levels of human perception and different scale and level of detail of urban environment. By linking the patterns of behavior and different levels of pedestrian activity with the knowledge how people percept and experience urban environment we focus on the links between them and try to model the non-tangible information that through its multilayered representation will indicate the interdependencies between patterns of pedestrian behavior, intensity of flows, visibility analysis and configuration of the streets in order to transform the whole system into the valuable tool for better comprehension of urban environment. The correspondence and interaction between different levels of pedestrian behavior should be enhanced with the selection of appropriate analytical tools that correspond to the level of activity that is explored. 


\subsection{Visibility analysis - assessment of surrounding through isovists field}

Visibility is predominant in the human perception of the urban fabric. The best illustration is [2] who notices that there were "many attempts to translate visual-perception research into architectural and urban design" and the visual appearance of cities. They assert that city mental maps contain many elements that can describe our experience and the image of the environment.

As noticed by [2], one way to link perceptual factors with spatial information is provided by the concept of isovist. An isovist is the set of all points in an environment of opaque surfaces that are visible from a given point (the limit of the isovist is an artificial one functioning something like a horizon in the absence of any other intervening surfaces). An isovist is a 2D horizontal slice through the field of view taken at eye height and parallel to the ground plane. The appeal of the concept is that isovists are an intuitively attractive way of thinking about a spatial environment, because they provide a description of the space 'from inside', from the point of view of individuals, as they perceive it, interact with it, and move through it [3]. By itself, isovist theory is neither a solely optical nor psychological description of visible space. But it is psychophysical by design, in that its unit of analysis - the isovist radial - has one endpoint defined by the eye of the observer and the other by a point in the environment [4]. This level of interaction between the pedestrian and the immediate built environment can be clearly referred to the operational level of pedestrian behavior.

To quantify space in a more systematic way, [5] suggests that the way in which we experience a space, and how we use it, is related to the interplay of isovists. Therefore, isovists field records a single isovist property for all locations in a given area. The underlying aim is to assess the way corresponding features vary through space. To achieve such an objective, he defines six different shape indicators strongly related to openness such as: the visible area and the visible perimeter, the occlusivity of the isovist (the accumulated lengths of edges of the isovist boundary that are not solid wall stopping the isovist), the variance and skewness of the function of radial distances, and, at last the circularity of the isovist (a compactness measure).

\subsection{Space syntax analysis for depicting spatial configuration}

Closely related to visibility analysis there is the configurational approach to the analysis of urban settlement, that was introduced as space syntax in the mid 80s [7].

So far, several operational techniques have been proposed and used, side by side with the original version. Yet, all the different techniques share some common elements, which were placed by Bill Hillier at the very root of the configurational approach [8]. Among the full set of the existing configurational techniques, by far the most known and used is the axial analysis, which reduces the urban grid into a set of segment, called lines, linking and covering all its spaces; the graphic representation of that system is called axial map. The most significant configurational indices (and those we are particularly interested in, in the following of the present paper) are connectivity and, integration.

Connectivity and integration (either global or local) indices are both first order measures. It is also possible to develop second order measures by correlating these first order measures. One of that is called intelligibility. It is the correlation between connectivity and integration. It describes how far the depth of a space from the layout as a whole can be inferred from the number of its direct connections, i.e. what can be understood of the global relation of a space from what can be observed within that space. In other words, if locally well connected lines are also integrating lines, then the correlation will be strong and the whole can be read from the parts. Conversely, if well connected lines are not also integrating lines, then the correlation will be poor and the whole will not be readable from the parts. A further configurational parameter that can be usefully analyzed in our case study is the so-called controllability value, which describes the way (and the intensity) a place in the grid is actually controlled from all the other locations. 
Usage, Usability, and Utility of 3D City Models

Table 1. Knowledge categories related to pedestrian movement.

\begin{tabular}{|c|c|}
\hline Type of knowledge & Knowledge category \\
\hline Environmental Factors & $\begin{array}{r}\text { Street type, Attractors, Integration, Visual fields, Level of service, } \\
\text { Level of comfort, Popularity }\end{array}$ \\
\hline Situational Factors & Traffic intensity, Season, Time of Day, Seasonal/daily Events \\
\hline Individual/Group & Stereotypical type, Preferences, Demographics, Visit frequency \\
\hline
\end{tabular}

\subsection{Urban knowledge related to pedestrian behavior}

A variety of urban context data (situational and environmental) and additional pragmatic factors were expected to play a prominent role in establishing appropriate context for our particular research of pedestrian movement. Some of these categories represent invariant properties of the urban context that can be obtained from the domain-specific urban knowledge, and the specifics of the particular scenario. In our case urban knowledge related to the environmental factors has been enriched by the results of the visibility analysis and configurational analysis establishing a productive link between different analytical tools and an appropriate level of pedestrian behavior resulting in a specific type of knowledge. Others should be drawn from the information gathered from a variety of sources (e.g., social studies, ambient and smart technologies). The set of selected categories could be broken down into three groups: (1) environmental factors, (2) situational factors reflecting the particularities of a given situation, (3) characteristics to account for the individual and group perspectives. The justification for the selection of the characteristics is based on the theory and empirical evidence reported in relevant literature.

\section{MODELING HUMAN SPATIAL BEHAVIOR}

\subsection{Case study: Old Bazaar in Skopje, Macedonia}

Skopje Old Bazaar is a mediaeval city fragment with a specific urban structure. The oldest inherited element of the Bazaar with un-doubtful authenticity is the street network layout. Over the centuries it didn't experience significant changes. Therefore, the streets are relatively small in size and informal in structure. However, the street network makes the very strong and comprehensible spatial network since its elements and their junctions rhythmically repeat within the system, creating a picture that resembles certain manifestations in the nature.

One of the traditional techniques of recording pedestrian activity, as an indicator of mobility flows, is recording the number of people passing through virtual points or 'gates' strategically positioned at the crossroads of the street network. The recording is usually conducted during several short periods of time in the course of the day. The spatial distribution of pedestrian movement intensity at different gates of the Old Bazaar (Figure 1) maps the pedestrian activity at tactical level. At the operational level pedestrians make instantaneous decisions for the immediate time period and the built environment around them whether it is based on its physical or configurational aspects. Decisions made on this level are recognized as the walking behavior of the pedestrians. This level brings up the interaction between the activity of the person, its spatial perception and its interaction with the urban environment. Several patterns have been revealed: (1) the highest intensity in pedestrian movement is recorded at the entrances of the Old Bazaar area; (2) the highest intensity of pedestrian movement is concentrated along the two main streets connecting the city center with the green market, which is located at the edge of the Old Bazaar; (3) most of the gates with low level of pedestrian movement are clustered and positioned in compact parts of the Old Bazaar.

The pedestrian choice to use certain streets within the urban protected areas besides depending on the morphology of the urban elements - the street network, the position of the attractors etc., will be influenced by the level of services as well as the level of comfort. It is also connected with the level 

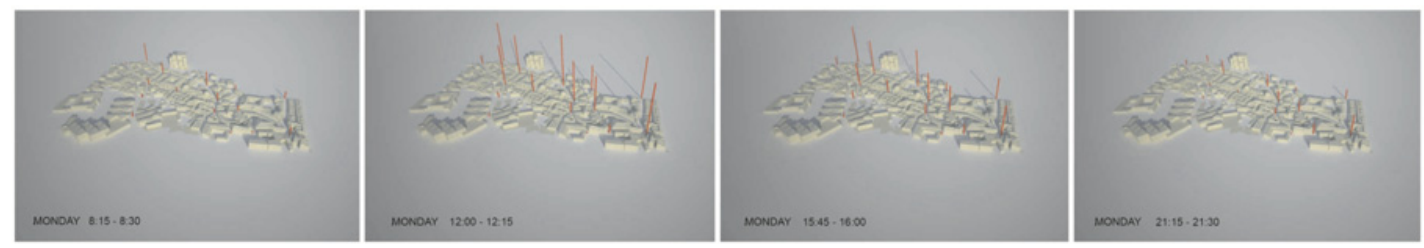

Figure 1. Spatial distribution of pedestrian movement within the area of Old Bazaar.

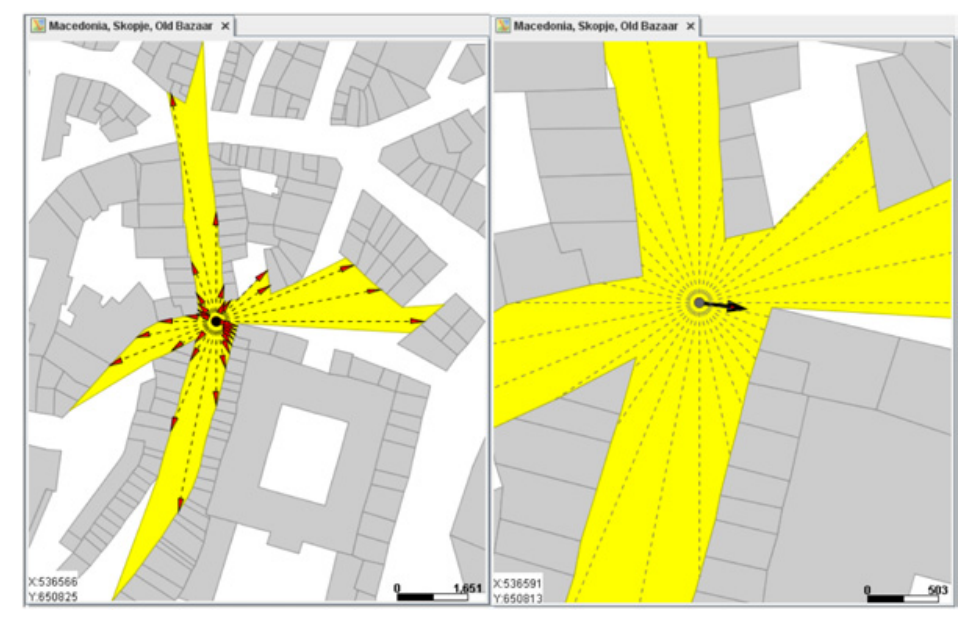

Figure 2. Bird eye view of an isovist in the Old Bazaar in Skopje. Buildings are represented by light gray polygons. The vantage point is represented by a black focal point. The corresponding isovist (urban viewshed) is the yellow colored polygon. 32 isovist radials are drawn (regularly spaced by an angle of $11.25^{\circ}$ ). The Euclidean vector represented on the right hand side figure corresponds to the visual drift at the given location.

of comfort provided with possibility for shelter, protection from weather, other traffic and crime. This pedestrian preference of points that are visually more open and connected to other parts of the route is confirmed with the analysis of the visual fields from all seventeen points - gates where a record of pedestrian movement has been performed.

\subsection{Isovist field measures}

According to [9], neurophysiologic data and behavioral theory suggest that enclosure is a very important property of the environment. One technique for measuring enclosure is the mathematical construct of an isovist. Redefined into space syntax theory, an isovist is "the sum of the infinite number of lines of sight (or axial lines) that pass through a single point in space (usually at eye height) and occupy the same plane (usually parallel to the ground plane)" [10]. If we assume that space is uniformly weighted from the generating location or viewpoint (we mean that each point of the isovist produces an equivalent visual stress towards the vantage point), the sum of all Euclidean vectors starting from vantage point towards all isovist's points corresponds to the local resulting visual stress (see Figure 2).

This spatial continuous Euclidean summation (integration) corresponds, by definition, to the Euclidean vector which starts from vantage point towards the center of gravity of the corresponding isovist. It also corresponds, by definition, to the drift mentioned in [11]. The isovist drift is a sort of accumulated visual stress all over the isovist shape. Therefore, it is not only a measure of the distance of the vantage point from the gravity centre of the corresponding isovist, but also a measure of the effort 


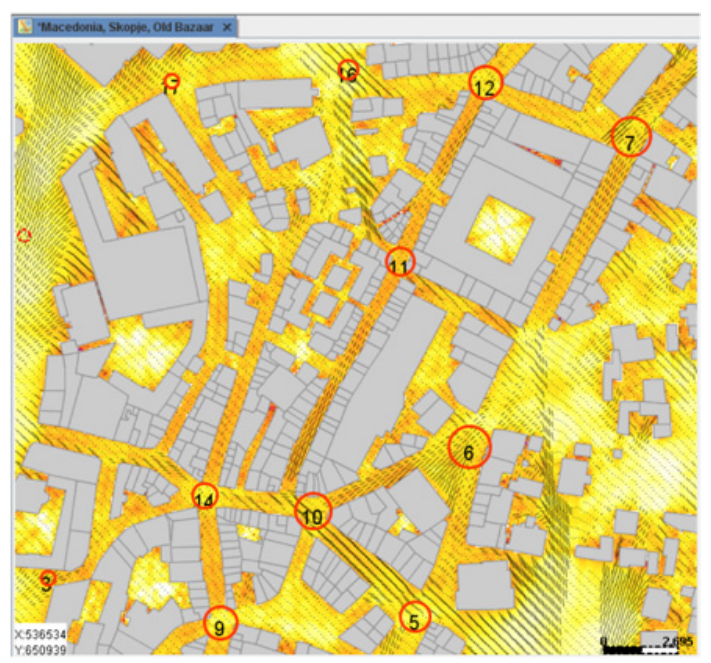

Figure 3. Representation of the drift Euclidean vector field superimposed to the Entropy of the function of radial distances in each given location (vantage point or isovist generating point).

required to align the visual center of a given open space (assuming isovist is an open space delineating solution) with its own center of gravity.

If drift can be seen as a sort of resultant force, that may mean there exists some sort of force field: a non contact forces field acting on a pedestrian at various positions in urban space. The influence of the force that the one would feel if he/she was at the given position. The isovists field representation exhibited in Fig. 3 sums all single visual perceptions in each vantage point and offers an objective and synthetic overview of a given urban fabric. More precisely, it combines two different shape indicators: a Shannon Entropy of the function of radial distances of the isovist in each point (the darker the background color is, the more predictable corresponding surroundings are), and - at the foreground a black colored drift vector.

Such a local assessment of the surrounding spatial configuration would obviously make the most from a real 3D approach. Nevertheless, concerning 3D aspects, one must admit that decision making process of a moving pedestrian integrates essentially the elevation gradient (which has probably more to do with the tactical level than with the operational one).

\subsection{Spatial configurations}

The axial analysis for the Old Bazaar has been done with the well known Depthmap software, available at the UCL space syntax website. Figure 4 shows the output of Depthmap as regards the configurational indices mentioned above.

The main qualitative outcomes of these indexes are:

- Connectivity in the Old Bazaar is quite high and uniformly distributed (with two hot-spots in the northern and southern parts). This means that, for a pedestrian, it is easy strolling in all the area, avoiding dead-end lines and unwanted detours;

- Compared to other case-studies, integration and connectivity maps look very similar (even if this fact is partly due to small size of the area). This means that the spatial configuration of the Bazaar is highly intelligible; and, as a consequence for tourists, the way-finding in the area is rather simple. In Figure 5 the distribution of controllability value in the grid of the Old Bazaar is chromatically represented, so as to highlight the urban places that meanly are actually easier to control from any 


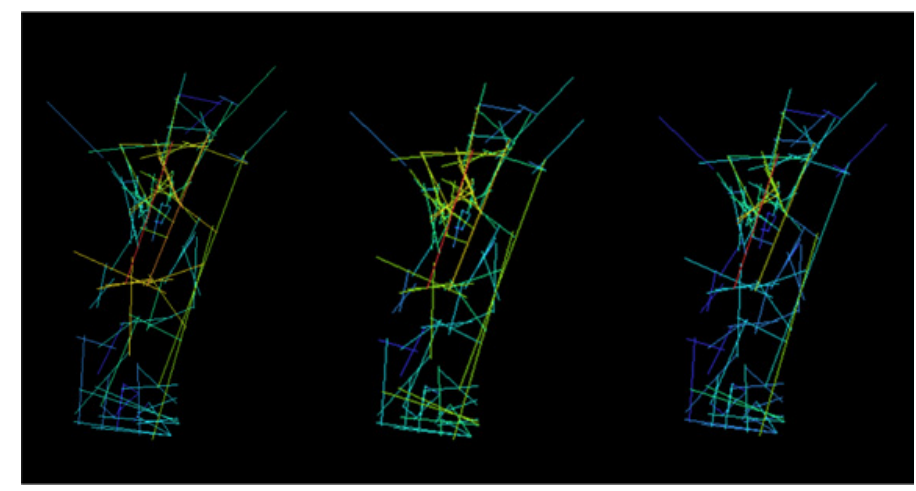

Figure 4. Spatial distribution of global integration, local integration and connectivity indices (left to right).

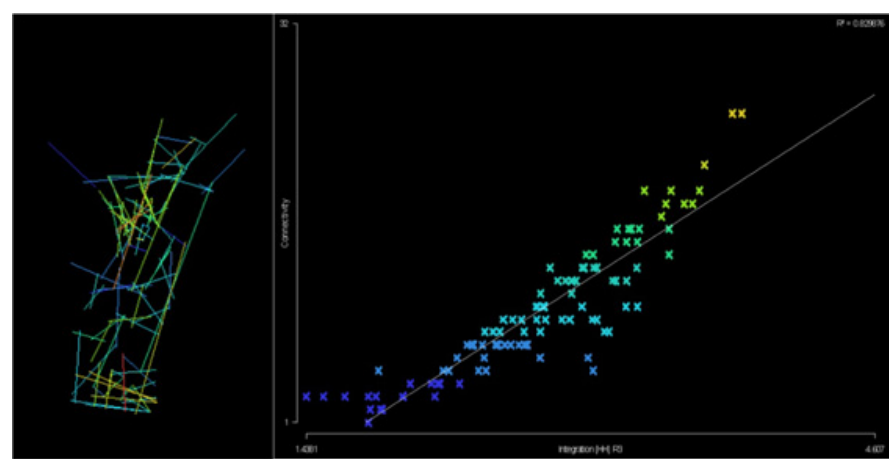

Figure 5. On the left, the spatial distribution of controllability index; on the right, the correlation of connectivity vs. local integration.

location. The two above mentioned hot-spots are this kind of locations; and, as a matter of facts, they are the preferred meeting points for tourists, confirming the controllability.

Beside, in the same figure the correlation analysis of connectivity value versus local integration value is presented: the outstanding R2 value that results over 0.83 definitively stands for the high level of intelligibility the grid of the old bazaar actually is provided with.

The configuration of the street network and the level of integration of the streets is not the only aspect of the built environment that determines the way people behave. However, it is the one that can be mapped and used along with other data as a tool for understanding the pedestrian mobility. The fact that the patterns of activity could change, regardless the fact that configuration of the streets rarely changes does not delegitimize the usefulness of this tool. This is the reason why this mapping of the level of integration methodology should be used in comparison and in combination with other types of data recording and research results.

\subsection{Knowledge integration}

Individual pieces of knowledge or a collection of categories do not constitute a model. The efforts to find out how those pieces of information are related to each other and affect pedestrian mobility are far more challenging. We argue that causal probabilistic models that are based on Bayesian inference provide a potential solution to some of the challenges in the pedestrian agent reasoning. It provides a formal method for quantifying uncertainty by combining diverse types of evidence including both 


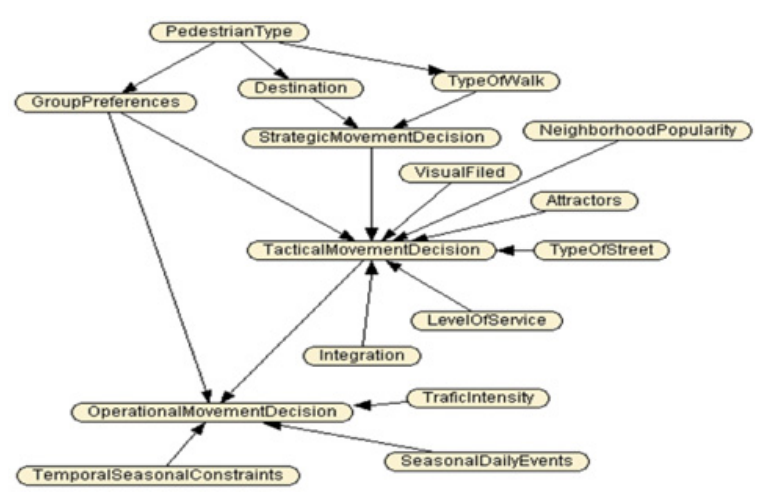

Figure 6. High-level dependencies between urban knowledge categories for pedestrian agent reasoning.

subjective beliefs (expert and participants) and objective data. Explanations of behavior derived from realistic causal model are more likely to match the reality, because the model accounts for deeper and richer relationships underneath data and simplistic statistical analysis.

Bayesian Belief Networks (BBNs) are suitable for representing the causal relationship between different pieces of information, as well as the rules for how to use, maintain, and reason with urbanrelated knowledge. The Bayesian network representing the agent's reasoning and decisions regarding pedestrian movement is shown in Figure 6. It should be noted the figure depicts variables and relations only on the highest level; in practice the network was quite complex. As shown, the agent movement decisions are related to the states of three hypothetical (non-observable) variables: Strategic Movement Decisions, Tactical Movement Decisions, and Operational Movement Decisions. Each of the hypothetical variables is linked to the relevant urban knowledge categories (drawn as oval boxes), which correspond to the categories discussed in previous Section.

Skopje's Old Bazaar, was the focus in our case study, a sample solution that may, by analogy, suggest ways to tackle problems that might be encountered in another context. Agents modeling have started by identifying different agent types to represent distinct entities and processes at play in the enclosed urban area. Several aggregated agent types were selected to represent different pedestrians at strategic level, namely, tourists, transit pedestrians, employees and visitors of various service providers (public, private). A number of realistic trajectories were collected from the history of pedestrian activity, which was used to establish the space of possible routes associated with aggregated pedestrian types. The visualization of the recorded pedestrian data has revealed several patterns with specific spatial and time distribution. It provided insight into both movement patterns and overall system behavior. A number of environmental factors that described the local context in terms of urban structure were explored in order to establish their impact and sensitivity during pedestrian route-finding decisions (tactical behavior). The overall integration value of the street network in the Old Bazaar indicates that highly-integrated streets are the ones in the middle of the area, while the peripheral streets have lower integration values. The exhibited pattern coincides well with the most frequented pedestrian routes. Data analysis has confirmed pedestrian preference for streets that are visually open and connected to the other parts of the route. The observed preference of wider non-deserted, though not overly crowded streets, has been considered.

The identification of attractors governing pedestrian dynamics within our context of interest was one of the objectives for gathering exhaustive context knowledge. Each urban entity or area, private and commercial, identified as a possible attractor or destination, were enriched by several context attributes such as type, functionality, ownership, working hours, demographics, number of employees, frequency of visitors, etc. The pedestrian routes and paths were described according to their accessibility, obstacles, sun exposure, street lights positions, dimensions, and throughput, in order to take into account how the characteristics of the real environment affect and constrain the pedestrian movement. Entities and 
areas outside the Old Bazaar perimeter were also included to account for the fact that their attraction, prominence and affordance may distinctively affect different agent type's behavior by extending the space of destinations. Street features, nearby attractor buildings, and time of the day were found to have significant impact on the movement preferences across the pedestrian types. Situational factors that characterize the particular scenario have been accounted for to simulate the exhibited behavior on an operational level. Description of above mentioned characteristics requires a 3D city model that will provide an appropriate tool for analysis of pedestrian routes and the impact of the inherently 3D environment to the pedestrian behavior at the operational level. Therefore, the knowledge gained through this complex and multilayered analysis of different aspects of the pedestrian mobility and the built environment, using various methods of data recoding and analysis of the activities and configurational nature of the urban environment with the use of $3 \mathrm{D}$ city models is essential for any future planning and designing activity.

\section{CONCLUSIONS}

The new integration of information and knowledge impose the greatest problem and raise many technical issues that are yet to be resolved. The integration of the interoperability of many different aspects of the spatial systems of cities raises the question of the unifying code that should provide us with the platform for performing such an operation. This also includes the agility of the model to shift between different scales and the level of detail of urban elements and urban systems and furthermore towards the exploration of integrated 3D spatial data, allowing us to become aware what the mutual influences that affect spatial developments are.

Use of visual analysis and agent-based models possess suitable capabilities required for modeling structural and behavioral properties of real-world urban systems, since the complex agencies are modeled and the behavioral properties of the agencies can be explicitly specified in terms of meaningful operations and applicable knowledge rules. Multi-agent models provide facilities for the management of spatio-temporal relations and cover features, such as interactions and mobility, which are imperative for modeling urban dynamics. Context-mediated approach for representation of agent reasoning mechanism has provided plausible modeling methodology based on insights and data analysis.

The diversity of our research team, comprised of architects, computer scientists and urban planners, reflects our acknowledgment of the interdisciplinary nature of the problem under consideration, which requires efforts that exceed the expertise of any individual field. The strength of integrating data and knowledge produced by various disciplines, apart from infusing the process with wellfounded methodologies, it also establishes a communication channel for negotiating conclusions and explanations that correspond more closely to the reality.

\section{References}

[1] Hoogendoorn, S.P. \& P.H.L. Bovy (2001), State-of-the-art of vehicular traffic flow modelling, Proceedings of the Institution of Mechanical Engineers - I 215(4), 283-303.

[2] E. Morello and C. Ratti, "A digital image of the city: 3D isovists in Lynch's urban analysis," Environment and Planning B: Planning and Design, vol. 36, no. 5, pp. 837-853, 2009.

[3] G. Weitkamp, "Capturing the view: a GIS based procedure to assess perceived landscape openness," Wageningen University, The Nederlands, 2010.

[4] A. Turner, M. Doxa, D. O'Sullivan, and A. Penn, "From isovists to visibility graphs: a methodology for the analysis of architectural space," Environment and Planning B: Planning and Design, vol. 28, no. 1, pp. 103-121, 2001.

[5] J. Wise, "NASA Contractor report 177501: The Quantitative Modelling of Human Spatial Habitability," Moffett Field, California, 1988. 
[6] M. L. Benedikt, "To take hold of space: isovists and isovist fields," Environment and Planning B: Planning and Design, vol. 6, no. 1, pp. 47-65, 1979.

[7] Hillier, B. \& Hanson, J. (1984) The Social Logic of Space, Cambridge: Cambridge University.

[8] Hillier, B. (1996) Space is the Machine. A configurational theory of architecture, Cambridge: Cambridge University Press.

[9] A. E. Stamps, "Isovists, enclosure, and permeability theory," Environment and Planning B: Planning and Design, vol. 32, no. 5, pp. 735-762, 2005.

[10] R. C. Dalton and S. Bafna, "The syntactical image of the city," in Proceedings of the 4th International Space Syntax Symposium, 2003, pp. 59.1-59.22.

[11] R. Conroy, "Spatial Navigation In Immersive Virtual Environments," The faculty of the built environment, University College London, London, U.K., 2001. 